\title{
How We Treat lung Cancer during SARS-Cov-2 (COVID-19) Pandemic in India?
}

Humankind is facing a unique challenge to its existence from the COVID-19 pandemic which has engulfed almost the entire globe, with more than 200 countries affected so far. The clear but unforeseen threat of this invisible enemy (SARS-CoV-2) will soon pose a major threat to the global economy, by destabilizing the social balance and health-care system. The motto of human life as of today is to "survive by any means" on this planet and therefore has to be our cumulative and coordinated battle against this invisible enemy. The danger is so frightening that even the very resourceful and powerful countries of the world have started crumbling under the burden of day-to-day increasing number of deaths and lack of adequate health-care resources which are falling short day by day to fight the infection.

Population of every stratum is at risk of being infected. The patients with cancer are more prone to infection during this pandemic. ${ }^{[1,2]}$ Therefore, the field of oncology is going to be under tremendous stress for dealing with the alarming situation in the coming days.

Lung cancer is the most common cancer worldwide among males and the most common cause of cancer-related mortality as per GLOBOCAN 2018 data. ${ }^{[3]}$ Every year, nearly 70,000 new lung cases are diagnosed in India. Lung cancer patients are at much higher risk of infection as they are already compromised for respiratory reserve due to the disease burden, smoking-related lung injury with coexisting chronic pulmonary disease, treatment (immune check-point or tyrosine kinase inhibitor [TKI])-related lung injury, and on the top of that the SARS-CoV-2 primarily involves the respiratory system. A very early report supports the aforementioned fact that lung cancer patients are at a high chance of getting infected even when not on any active treatment. ${ }^{[1,4]}$ All oncologists around the globe are making standard operating procedures (SOP) to optimize the cancer treatment during this pandemic with the following aims - (a) To decrease the burden on already overstretched health-care resources by reducing treatment-related toxicities, (b) to weigh the risk against benefit of any oncological intervention in the context of this pandemic for reducing the risk of infection and resultant morbidity-mortality, (c) to decrease the unnecessary footfall in hospital settings for merely follow-up and review of cancer patients who are not on any active treatment and thus reducing community spread of the infection, and (d) to delay or defer any oncological treatment in case of slow-growing disease where immediate treatment is not warranted.

Hence, the basic questions are - (a) Which patients should get immediate treatment?, (b) in which cases can the treatment be delayed or deferred without compromising the oncological outcome?, and (c) which patients should not be offered any active treatment and should remain on best supportive care because social distancing overweighs the risk of getting COVID-19 infection.

We have reviewed the recommendations of NCCN, ASCO, ESMO, and IASLC for systemic therapy; and landmark randomized control trials for guiding systemic therapy in lung cancer patients during this pandemic keeping in mind the risk-benefit ratio of any intervention, logistics of access to care including transport, and the availability of resources.

All lung cancer patients, especially those with localized disease where multidisciplinary management is mandated, should be discussed in multidisciplinary tumor board in which a clinical microbiologist or infectious disease specialist should also preferably be included in the current context.

All medical oncologists adopting the following approach must also use their own judgment on a case-to-case basis and must follow local institutional SOP, if any, in the context of COVID-19 pandemic.

\section{Non-small Cell Lung Cancer}

\section{Localized resectable disease}

Localized resectable lung cancer (stage I to selected stage III) patients should be taken for upfront surgical resection, if deemed fit for surgery. Neoadjuvant chemotherapy can be detrimental as few patients may progress during chemotherapy as usual, and the chance of severe SARS-CoV-2 infection will be high due to expected myelosuppression during chemotherapy.

Adjuvant chemotherapy can be deferred for those with Stage IB disease due to the limited benefit of chemotherapy in this setting. For those with resected Stage IIA to Stage III (Stage IIIA and selected Stage IIIB), adjuvant chemotherapy should be given in patients without epidermal growth factor receptor (EGFR) mutation, if fit and can be replaced with adjuvant TKI, if they are found to be EGFR mutated. ${ }^{[5]}$ The usual adjuvant chemotherapy is a cisplatin-based doublet chemotherapy up to a maximum of four cycles.

\section{Unresectable Stage III Non-Small Cell Lung Cancer}

All fit patients should be treated with chemoradiotherapy (CTRT), whenever feasible, after symptom optimization and discussion in a tumor board meeting. Concurrent CTRT should be preferred over 
sequential CTRT in suitable patients in view of the survival benefit. ${ }^{[6]}$ Consolidation with durvalumab, an anti-PDL1 immune checkpoint inhibitor (ICI), should be considered in those with nonprogressive disease and $\leq$ grade 1 radiation pneumonitis after CTRT (both sequential and concurrent) due to significant survival advantage, though there is a potential danger of drug-induced pneumonitis and cytokine storm if infected with SARS-CoV-2. A high index of suspicion should be kept for SARS-CoV-2 for any new-onset cough or radiologic pneumonitis as the differential can be COVID-19, radiation pneumonitis, or ICI-induced pneumonitis.

\section{Advanced/metastatic disease}

a. EGFR-mutated non-small cell lung cancer (NSCLC): Anti-EGFR TKI should be the treatment of choice due to its nonmyelosuppressive nature, better tolerability, and superior outcome compared to cytotoxic chemotherapy. The second-generation (dacomitinib and afatinib) and the third-generation (osimertinib) TKI is preferred over the first-generation TKI due to greater efficacy. Among them, osimertinib remains the drug of choice. Few of them can cause drug-induced interstitial pneumonitis but usually of low grade and less in incidence. The combination of chemotherapy ${ }^{[7]}$ or antivascular endothelial growth factor agents ${ }^{[8]}$ with EGFR TKI should be avoided in the current context owing to their greater toxicity and more myelosuppression with the chemo-TKI combinations

b. ALK/ROS1 rearranged NSCLC: This subgroup of patients should be treated with TKI in a similar manner like EGFR-mutated lung cancer. ALK/ROS1 inhibitors are well-tolerated agents with very good safety profile and greater efficacy in ALK/ROS1-rearranged NSCLC, and hence, anti-ALK/ROS1 TKI should be the only agents to treat this groups of patients. ${ }^{[9]}$ Chemotherapy should be avoided, whenever feasible.

c. NSCLC without any driver mutation: NSCLC with PDL1 tumor proportion score (TPS) by DAKO $22 \mathrm{C} 3 \geq 50 \%$ should be treated with single-agent pembrolizumab, wherever applicable due to high cost of therapy. ${ }^{[10]}$ Adenocarcinoma with TPS 1\%-49\% can be treated by either 4 cycles of pemetrexed/ platinum doublet, followed by pembrolizumab maintenance $^{[11]}$ (thus avoiding initial chemo-ICI combination therapy in view of higher toxicity) or with single-agent pembrolizumab as per KEYNOTE 042 study. ${ }^{[12]}$ The pemetrexed maintenance strategy for responders to chemotherapy can be delayed in view of minimal survival benefit and reducing day-care footprint. Adenocarcinoma with negative PD-L1 score may be treated by pemetrexed/platinum-based therapy for 4 cycles, followed by optional pemetrexed maintenance. This compromise on survival weighs greater than the risk of acquiring SARS-CoV-2 infection. Optional pembrolizumab maintenance should again can be deferred due to the limited efficacy in PD-L1 in negative cohort. ${ }^{[1]}$ Those with squamous cell histology can be treated with chemo-ICI combination ${ }^{[13]}$ or with 4 cycles of platinum-based doublet, followed by pembrolizumab (ICI) maintenance, thus avoiding initial higher toxicity of chemo-ICI combination

d. NSCLC with rare driver mutation: Those with BRAF $\mathrm{v} 600 \mathrm{e} / \mathrm{k}$ mutation can be treated with BRAF-MEK inhibitor combinations, such as dabrafenib and trametinib, which are less toxic than chemotherapy and MET Exon 14 skipping mutation or high MET amplification can be treated with MET inhibitor such as-crizotinib, and tepotinib.

\section{Small-Cell Lung Cancer}

a. Limited stage disease: It should be treated with CTRT in a fit patient in view of the aggressive nature of small cell carcinoma of the lung. Prophylactic cranial irradiation (PCI) can be deferred by 6-8 weeks.

b. Extensive stage disease: Patients with Eastern Cooperative Oncology Group (ECOG) performance status (PS) of 0 and 1 can be treated with a total of 4 cycles of platinum (preferably cisplatin due to less myelosuppression) and etoposide-based doublet chemotherapy with liberal use of growth factor. For PS 2 patients, either single-agent carboplatin or best supportive care may be the treatment of choice. PCI should not be given to patients with extensive stage disease irrespective of treatment response. Chemo-ICI combination (etoposide, platinum, and atezolizumab) ${ }^{[14]}$ can be modified to 4 cycles of platinum doublet, followed by atezolizumab maintenance as the response rate is not much different with chemo versus chemo-ICI combination.

\section{Lung Cancer Clinical Trial Patients}

Every effort should be made to maintain the protocol adherence in all lung cancer patients under different clinical trials in lung cancer as the scientific value of such research is enormous for the field of medical oncology as well as for millions of future lung cancer patients. However, at the same time, patients' safety should be assessed first, and the whole clinical trial team should take a collective decision whether to continue therapy as per schedule or to defer the scheduled therapy, if not harmful to the patients. Screening and enrollment of new patients should be kept on hold during this COVID-19 epidemic.

\section{Lung Cancer Patients on Follow-up}

Those who have completed their initial treatment and are on routine follow-up can be consulted over telephone or through telemedicine to enquire and assure them about their disease with simple instructions, to inform and educate them about do's and don'ts during COVID-19, and to reschedule their appointment in a later appropriate date. 
Every attempt should be made to decrease unnecessary and unwarranted hospital visits. They should visit their family physician or local physician for any nononcology-related problems and necessary prescriptions.

\section{Personal Protection, Routine Vaccination, and Comorbidity Control}

All patients and their caregivers should maintain personal hygiene, adopt protective measures, and maintain social distancing as per the guidelines laid down by the local and national health authorities. Routine vaccination, such as yearly influenza vaccine and pneumococcal vaccine should be continued, if feasible locally without affecting the personal protection. Patients should take extra precaution by frequent hand-washing and strict social distancing from sick family members and self-quarantine themselves, if symptomatic. Those with chronic obstructive pulmonary disease should be advised to use inhalers through a spacer device, and nebulizers must be discouraged.

To conclude, all localized lung cancer patients should be discussed in multidisciplinary tumor board, and treatment decision should be made after an informed decision process involving patients and their caregivers keeping in mind the availability of local health-care resources. Clear judgment needs to be made by the treating team keeping in mind the risk-benefit profile of any intervention. For the treatment of advanced/metastatic disease, every effort should be made to detect any driver mutation by appropriate molecular analysis as TKI is safer than chemotherapy and/or ICI during this pandemic. Combination therapy of chemotherapy and/or biologics or ICI can be sequenced in a customized manner just to avoid excess toxicity, and pemetrexed maintenance may be kept as optional during this pandemic. Patients of advanced NSCLC without any driver mutation and extensive stage SCLC with ECOG PS $\geq 2$ should be offered palliative care. Any lung cancer patients on treatment or on follow-up visiting casualty with shortness of breath should be suspected of having infection with of SARS-CoV-2 until and unless proven otherwise, as there are many confounders - many patients with COVID-19 who may not have fever ${ }^{[15]}$ Such lung cancer patients may have existing chronic pulmonary disease, pleural effusion, drug (ICI) and radiation-induced pneumonitis, bacterial pneumonia, and common flu infection. Threshold for the treatment of lung cancer patients should be kept high in those with multiple comorbidities and age $>60$ years, as the case fatality is the highest in this group. ${ }^{[4,15]}$

\section{Bivas Biswas', Sandip Ganguly', Deepak Dabkara', Joydeep Ghosh', Priyanka Srivastava ${ }^{2}$, Prashant Mehta ${ }^{3}$, Amol Patel ${ }^{4}$, Sainath Bhethanabhotla ${ }^{5}$, Atul Batra ${ }^{6}$, Raja Pramanik ${ }^{6}$, Vineet Govinda Gupta ${ }^{7}$, Chandan Krushna Das ${ }^{8}$, Shubh Mahindru ${ }^{9}$}

${ }^{\prime}$ Department of Medical Oncology, Tata Medical Center, Kolkata, West Bengal, India, ${ }^{2}$ M.S. Patel Cancer Center, Shreekrishna Hospital and Research Center, Karamsad, Gujarat, India, ${ }^{3}$ Department of Medical Oncology/Hematoncology and BMT, Asian Institute of Medical Sciences, Faridabad, Haryana, India, ${ }^{4}$ Department of Medical Oncology, Army Hospital Research and Referral, New Delhi, India, ${ }^{5}$ Department of Medical Oncology, Care Cancer Institute, Hyderabad, Telangana,

India, ${ }^{6}$ Department of Medical Oncology, Care Hospitals, All India Institute of Medical Sciences, New Delhi, India, ${ }^{7}$ Department of Medical Oncology, Artemis Hospital, Gurgaon, Haryana, India, ${ }^{8}$ Regional Cancer Center, Post Graduate Institute of Medical Education and Research, Chandigarh, India, ' Department of Surgical Oncology, Ivy Hospital, SAS Nagar, Punjab, India

Address for correspondence: Dr. Bivas Biswas, Department of Medical Oncology, Tata Medical Center, Kolkata, West Bengal, India. E-mail: bivasbiswas@gmail.com

Submitted: 01-Apr-2020 Accepted in Revised Form: 13-Apr-2020 Published: 08-May-2020

\section{References}

1. Onder G, Rezza G, Brusaferro S. Case-fatality rate and characteristics of patients dying in relation to COVID-19 in Italy. JAMA 2020 Mar 23. doi: 10.1001/jama.2020.4683. Online ahead of print.

2. Wang H, Zhang L. Risk of COVID-19 for patients with cancer. Lancet Oncol 2020;21:e181.

3. Globocan 2018: India Factsheet. India against Cancer; 2018. Available from: http://cancerindia.org.in/globocan-2018india-factsheet/. [Last accessed on 2020 Apr 01].

4. Yu J, Ouyang W, Chua MLK, Xie C. SARS-CoV-2 transmission in patients with cancer at a tertiary care hospital in Wuhan, China. JAMA Oncol 2020 Mar 25;e200980. doi: 10.1001/ jamaoncol.2020.0980. Online ahead of print.

5. Tang W, Li X, Xie X, Sun X, Liu J, Zhang J, et al. EGFR inhibitors as adjuvant therapy for resected non-small cell lung cancer harboring EGFR mutations. Lung Cancer 2019;136:6-14.

6. Curran WJ Jr., Paulus R, Langer CJ, Komaki R, Lee JS, Hauser S, et al. Sequential vs. concurrent chemoradiation for stage III non-small cell lung cancer: Randomized phase III trial RTOG 9410. J Natl Cancer Inst 2011;103:1452-60.

7. Noronha V, Patil VM, Joshi A, Menon N, Chougule A, Mahajan A, et al. Gefitinib versus gefitinib plus pemetrexed and carboplatin chemotherapy in EGFR-mutated lung cancer. J Clin Oncol 2020;38:124-36.

8. Saito H, Fukuhara T, Furuya N, Watanabe K, Sugawara S, Iwasawa $\mathrm{S}$, et al. Erlotinib plus bevacizumab versus erlotinib alone in patients with EGFR-positive advanced non-squamous non-small-cell lung cancer (NEJ026): Interim analysis of an open-label, randomised, multicentre, phase 3 trial. Lancet Oncol 2019;20:625-35.

9. Patel A, Batra U, Prasad KT, Dabkara D, Ghosh J, Sharma M, et al. Real world experience of treatment and outcome in ALK-rearranged metastatic nonsmall cell lung cancer: A multicenter study from India. Curr Probl Cancer 2020 Mar 17;100571. doi: 10.1016/j.currproblcancer.2020.100571.

10. Reck M, Rodríguez-Abreu $\mathrm{D}$, Robinson AG, Hui R, Csőszi T, Fülöp A, et al. Pembrolizumab versus chemotherapy for PD-L1-positive non-small-cell lung cancer. N Engl J Med 2016;375:1823-33.

11. Gandhi L, Rodríguez-Abreu D, Gadgeel S, Esteban E, Felip E, De Angelis F, et al. Pembrolizumab plus chemotherapy in metastatic non-small-cell lung cancer. $\mathrm{N}$ Engl $\mathrm{J}$ Med 
2018;378:2078-92.

12. Mok TSK, Wu YL, Kudaba I, Kowalski DM, Cho BC, Turna HZ, et al. Pembrolizumab versus chemotherapy for previously untreated, PD-L1-expressing, locally advanced or metastatic non-small-cell lung cancer (KEYNOTE-042): A randomised, open-label, controlled, phase 3 trial. Lancet 2019;393:1819-30.

13. Paz-Ares L, Luft A, Vicente D, Tafreshi A, Gümüş M, Mazières $\mathrm{J}$, et al. Pembrolizumab plus chemotherapy for squamous non-small-cell lung cancer. $\mathrm{N}$ Engl $\mathrm{J}$ Med 2018;379:2040-51.

14. Horn L, Mansfield AS, Szczęsna A, Havel L, Krzakowski M, Hochmair MJ, et al. First-line atezolizumab plus chemotherapy in extensive-stage small-cell lung cancer. $\mathrm{N}$ Engl $\mathrm{J}$ Med 2018;379:2220-9.

15. Guan WJ, Ni ZY, Hu Y, Liang WH, Ou CQ, He JX, et al. Clinical characteristics of coronavirus disease 2019 in China. N Engl J Med 2020 Feb 28;NEJMoa2002032. doi: 10.1056/ NEJMoa2002032. Online ahead of print.
This is an open access journal, and articles are distributed under the terms of the Creative Commons Attribution-NonCommercial-ShareAlike 4.0 License, which allows others to remix, tweak, and build upon the work non-commercially, as long as appropriate credit is given and the new creations are licensed under the identical terms.

For reprints contact: WKHLRPMedknow_reprints@wolterskluwer.com

\begin{tabular}{|l|l|}
\hline \multicolumn{2}{|c|}{ Access this article online } \\
\hline Quick Response Code: & Website: \\
& www.ijmpo.org \\
\cline { 2 - 2 } & DOI: \\
\hline
\end{tabular}

How to cite this article: Biswas B, Ganguly S, Dabkara D, Ghosh J, Srivastava $P$, Mehta $P$, et al. How we treat lung cancer during SARS-Cov-2 (COVID-19) pandemic in India? Indian J Med Paediatr Oncol 2020;41:149-52. 